PROCEEDINGS OF THE

AMERICAN MATHEMATICAL SOCIETY

Volume 126, Number 4, April 1998, Pages 1031-1042

S 0002-9939(98)04092-1

\title{
SECOND ORDER CONFORMAL COVARIANTS
}

\author{
THOMAS BRANSON \\ (Communicated by Palle E. T. Jorgensen)
}

\begin{abstract}
We complete the classification of second order, bundle valued differential operator invariants of Riemannian and pseudo-Riemannian conformal structure, by classifying such operators which pass between bundles associated to different representations of the rotation or spin group.
\end{abstract}

\section{INTRODUCTION}

A conformal covariant is a universal formula which assigns to each $n$-dimensional Riemannian manifold $(M, g), n \geq 3$, a linear differential operator $D: \mathbb{V} \rightarrow \mathbb{W}, \mathbb{V}$ and $\mathbb{W}$ being natural vector bundles, which responds to local metric rescalings by

$$
\bar{g}=\Omega^{2} g, 0<\Omega \in C^{\infty}(M) \quad \Longrightarrow \quad \bar{D} \varphi=\Omega^{-b} D\left(\Omega^{a} \varphi\right),
$$

where $\varphi \in C^{\infty}(M, \mathbb{V})$. Since this statement is local, $D$ must be built locally from the metric $g$. For oriented Riemannian or Riemannian spin manifolds, the definition is the same, except that the volume form $E$ and/or fundamental tensor-spinor $\gamma$ may enter in the formula for $D$, and should scale in a way compatible with the scaling of $g: \bar{E}=\Omega^{n} E, \bar{\gamma}=\Omega^{-1} \gamma$. The best-known conformal covariant is the conformal Laplacian $\Delta+(n-2) K / 4(n-1)$ on ordinary functions, $\Delta$ being the Laplacian and $K$ the scalar curvature of $g$. Many more examples are known [4], [13], [5], [17], and important strides have been made in the direction of the general classification problem [1], [2], [14].

To classify conformal covariants, it is clearly sufficient to classify those which act between bundles which are irreducible under their structure group: $\mathrm{O}(n), \mathrm{SO}(n)$, or $\operatorname{Spin}(n)$. Since the problem is local, we may assume $\operatorname{Spin}(n)$ is the structure group. Since $\operatorname{Spin}(n)$ is connected and simply connected, $\operatorname{Spin}(n)$ equivariance is the same as $\mathfrak{s o}(n)$ (infinitesimal) equivariance. In [6, Sec. 5], we classified second order conformal covariants whose source and target are the same irreducible $\operatorname{Spin}(n)$ bundle. Here we complete the task by classifying second order conformal covariants with different source and target bundles.

Since the finite dimensional representation theory of $\operatorname{Spin}(p, q), p+q=n$, is the same (by the Weyl unitary trick) as that of $\operatorname{Spin}(n)$, we obtain, without further effort, a classification of second order covariants of pseudo-Riemannian conformal structure. The operators produced have the same expressions in invariant index notation, since this is signature independent. For example, the conformal Laplacian

Received by the editors September 3, 1996.

1991 Mathematics Subject Classification. Primary 47F05.

Research partially supported by NSF grant INT-9114401.

(C)1998 American Mathematical Society 
is $-\nabla^{i} \nabla_{i}+(n-2) R^{i j}{ }_{i j} / 4(n-1)$, and the Dirac operator is $\gamma^{i} \nabla_{i}$, regardless of the metric signature. To avoid circumlocution, we shall work below in the Riemannian $(\operatorname{Spin}(n))$ case.

To dodge complicated trivialities, the following conventions are needed. Second order will mean universally second order: to qualify as second order, an operator needs to be second order for any choice of the metric $g$. Thus, for example, the conformal Laplacian is second order, but $K \Delta$ is not (since $K$ vanishes for some choices of $g$ ). In addition, we shall work modulo actions of the Weyl conformal curvature tensor $C$ (see Remarks 2.4 and 3.1 below). If $D: \mathbb{V} \rightarrow \mathbb{W}$ is a second order conformal covariant, it may well happen that one or more isomorphic copies of $\mathbb{W}$ appear in $\mathcal{C} \otimes \mathbb{V}, \mathcal{C}$ being the bundle of algebraic Weyl tensors. If $\alpha \in$ $\operatorname{Hom}_{\mathfrak{s o}(n)}(\mathcal{C} \otimes \mathbb{V}, \mathbb{W})$, then

$$
\varphi \rightarrow D \varphi+\alpha(C \otimes \varphi)
$$

is also a conformal covariant. Our convention will be to assemble the operators (1.2), for fixed $D$, into one equivalence class, and count this class as one conformal covariant.

To state the old and new results, we need a classification of the bundles involved. Irreducible representations of $\operatorname{Spin}(n)$ are in one-to-one correspondence with dominant weights: tuples $\lambda$ in $\mathbb{Z}^{\ell} \cup\left(\frac{1}{2}+\mathbb{Z}\right)^{\ell}$, where $\ell=[n / 2]$, with [15]

$$
\begin{array}{ll}
\lambda_{1} \geq \lambda_{2} \geq \ldots \lambda_{\ell} \geq 0, & n \text { odd }, \\
\lambda_{1} \geq \lambda_{2} \geq \ldots\left|\lambda_{\ell}\right|, & n \text { even. }
\end{array}
$$

The dominant weight $\lambda$ is used to label the representation $\left(\lambda, V_{\lambda}\right)$ with highest weight $\lambda$. The representations which factor through $\mathrm{SO}(n)$ are exactly those with integral highest weights.

If $\mathcal{S}$ is the bundle of spin frames, we may form the associated vector bundle $\mathbb{V}(\lambda):=\mathcal{S} \times_{\lambda} V_{\lambda}$. For example, the cotangent (or tangent) bundle is associated with the defining representation of $\mathrm{SO}(n)$, and labelled by $\lambda=(1,0, \ldots, 0)$. The spinor bundle $\Sigma$ is labelled by $\left(\frac{1}{2}, \ldots, \frac{1}{2}\right)$ if $n$ is odd, and is the direct sum of the bundles labelled by $\left(\frac{1}{2}, \ldots, \frac{1}{2}, \pm \frac{1}{2}\right)$ if $n$ is even. Since the spinor representation is faithful, the set of $\operatorname{Spin}(n)$ bundles is the same as the set of irreducible summands of tensor-spinor bundles (though a given bundle always has infinitely many different tensor-spinor realizations). Thus our study of abstract $\operatorname{Spin}(n)$ bundles is equivalent to the study of tensor-spinor bundles.

A further labelling of bundles is possible if we pay attention to the effect of fiberwise dilation: enlarge the structure group to $H:=\operatorname{Spin}(n) \times \mathbb{R}_{+}$; the irreducible representations of $H$ have the form

$$
\lambda^{r}(m, a):=a^{r} \lambda(m), \quad r \in \mathbb{C}, m \in \operatorname{Spin}(n), a \in \mathbb{R}_{+} .
$$

We shall call the associated vector bundle $\mathbb{V}^{r}(\lambda) ; r$ is called the conformal weight. For example, $\mathbb{V}^{r}(0, \ldots, 0)$ is the bundle of $r / n$-densities; the cotangent bundle is $\mathbb{V}^{1}(1,0, \ldots, 0)$, and the tangent bundle is $\mathbb{V}^{-1}(1,0, \ldots, 0)$. If $D: \mathbb{V}^{r}(\lambda) \rightarrow \mathbb{V}^{s}(\mu)$ is conformally covariant of biweight $(a, b)$ (i.e. (1.1) holds), an easy calculation shows that we can get a conformal covariant of any other biweight $\left(a^{\prime}, b^{\prime}\right)$ by forgetting the extra structure to obtain $D: \mathbb{V}(\lambda) \rightarrow \mathbb{V}(\mu)$, then reweighting to obtain $D$ : $\mathbb{V}^{r-\left(a^{\prime}-a\right)}(\lambda) \rightarrow \mathbb{V}^{s-\left(b^{\prime}-b\right)}(\mu)$. In particular, by choosing the correct $\left(a^{\prime}, b^{\prime}\right)$ we can arrange for $D$ to be conformally invariant: $\bar{D}=D$. In another direction, we can 
define the reduced conformal biweight of a conformal covariant to be its biweight as an operator $\mathbb{V}^{0}(\lambda) \rightarrow \mathbb{V}^{0}(\mu)$.

Fegan [9] made the important observation that the gradients introduced by Stein and Weiss in [15] are conformally covariant. If $\nabla$ is the Riemannian spin connection, then

$$
\nabla: \mathbb{V}(\lambda) \rightarrow T^{*} M \otimes \mathbb{V}(\lambda) \cong_{\operatorname{Spin}(n)} \mathbb{V}\left(\sigma_{1}\right) \oplus \ldots \oplus \mathbb{V}\left(\sigma_{N}\right),
$$

where $N=N_{\lambda}$. The decomposition into irreducibles on the right has multiplicity one, and a given dominant weight $\sigma$ appears among the $\sigma_{u}$ if and only if

$$
|\sigma-\lambda|=1, \quad \text { or } \quad n \text { is odd and } \sigma=\lambda .
$$

Note that $|\sigma-\lambda|=1$ if and only if $\sigma=\lambda \pm e_{a}$, where $e_{a}$ is the $a^{\underline{\text { th }}}$ standard basis vector $(0, \ldots, 1, \ldots, 0)$. The gradient $G_{u}=G_{\lambda \sigma_{u}}$ is the composition $\operatorname{Proj}_{\sigma_{u}} \circ \nabla$. Fegan showed that $G_{u}$ has reduced conformal biweight $\left(p_{u}, p_{u}+1\right)$, where

$$
p_{u}=\frac{1}{2}\left(n-1+\left\langle\lambda+\sigma_{u}+2 \rho, \lambda-\sigma_{u}\right\rangle\right) .
$$

Here $2 \rho=(n-2, n-4, \ldots, n-2 \ell)$ is the sum of positive $\mathfrak{s o}(n)$ roots. By noticing that the formal adjoint of a conformal covariant is a conformal covariant, or by noticing that the formal adjoint of a gradient is a gradient, it is easy to show that $G_{u}^{*}$ has reduced conformal biweight $\left(n-1-p_{u}, n-p_{u}\right)$.

Theorem $1.1[6]$. Up to a constant multiple, there is a unique second order conformal covariant $D_{\lambda}: \mathbb{V}(\lambda) \rightarrow \mathbb{V}(\lambda)$ when $n$ is odd and $\lambda_{\ell} \neq \frac{1}{2}$, and when $n$ is even and $\lambda_{\ell}=0$. Otherwise there is no second order conformal covariant $\mathbb{V}(\lambda) \rightarrow \mathbb{V}(\lambda)$.

$$
D_{\lambda}:=\frac{K}{2(n-1)}-\sum_{u=1}^{N_{\lambda}}\left(p_{u}-\frac{n-2}{2}\right)^{-1} G_{u}^{*} G_{u}
$$

unless $n$ is even and $\lambda_{\ell}=0 \neq \lambda_{\ell-1}$, in which case $D_{\lambda}=G_{\lambda, \lambda \pm e_{\ell}}^{*} G_{\lambda, \lambda \pm e_{\ell}}$. The reduced conformal biweight of $D_{\lambda}$ is $((n-2) / 2,(n+2) / 2)$.

In the case where $n$ is odd and $\lambda_{\ell} \geq 1$, we were unable to prove in [6] that (1.5) describes the second order conformal covariant whose existence is asserted in the theorem's first sentence. (What could go wrong, a priori, is that (1.5) might be the zero operator, or have order zero.) This loose end was tied up in [7, Corollary 5.3].

The principal result of this paper is:

Theorem 1.2. Let $\lambda$ and $\mu$ be distinct dominant $\operatorname{Spin}(n)$ weights. There is a unique (up to a constant factor) second order conformal covariant $D_{\lambda \mu}: \mathbb{V}(\lambda) \rightarrow$ $\mathbb{V}(\mu)$ if and only if $\mu=\lambda+2 \varepsilon e_{a}$ for some $\varepsilon \in\{ \pm 1\}$ and $a \in\{1, \ldots, \ell\}$; otherwise there is no second order conformal covariant $\mathbb{V}(\lambda) \rightarrow \mathbb{V}(\mu)$.

$$
D_{\lambda \mu} \varphi=G_{\lambda+\varepsilon e_{a}, \mu} \varphi G_{\lambda, \lambda+\varepsilon e_{a}}+\operatorname{Proj}_{\mathbb{V}(\mu)} V \otimes \varphi=\operatorname{Proj}_{\mathbb{V}(\mu)}(\nabla \nabla+V \otimes) \varphi,
$$

where

$$
V:=\frac{1}{n-2}\left(\operatorname{Ric}-\frac{K g}{2(n-1)}\right),
$$

Ric being the Ricci tensor. The reduced conformal biweight of $D_{\lambda \mu}$ is

$$
\left(\frac{1}{2}\left(n-2 \varepsilon(\mu+\rho)_{a}\right), \frac{1}{2}\left(n-2 \varepsilon(\lambda+\rho)_{a}\right)\right) .
$$




\section{Proof of Theorem 1.2}

Throughout this section, we assume that $\mu \neq \lambda$. A first step is to classify the $H$-equivariant second order differential operators $\mathbb{V}(\lambda) \rightarrow \mathbb{V}(\mu)$; these form a vector space. The dimension of the space of $\operatorname{Spin}(n)$-invariant second order leading symbols is bounded by

$$
\operatorname{dim} \operatorname{Hom}_{\operatorname{Spin}(n)}\left(T^{*} M \otimes T^{*} M \otimes \mathbb{V}(\lambda), \mathbb{V}(\mu)\right) .
$$

This is in turn bounded by 2 , since there are at most two paths $\lambda \rightarrow \sigma \rightarrow \mu$ under the selection rule (1.3). In fact, it will turn out that there is at most one linearly independent $\operatorname{Spin}(n)$-invariant second order leading symbol.

Lemma 2.1. $h=h_{\lambda \mu}:=\operatorname{dim} \operatorname{Hom}_{\operatorname{Spin}(n)}\left(T^{*} M \otimes T^{*} M \otimes \mathbb{V}(\lambda), \mathbb{V}(\mu)\right)$ is nonzero only in the following cases.

Case I. $\mu=\lambda \pm 2 e_{a}$ for some $a$. Then $h=1$.

Case II. $\mu=\lambda \pm e_{a} \pm^{\prime} e_{b}$ (independent plus or minus signs), $a<b$. Then (case IIa) $h=2$ unless (case IIb) $b=a+1, \lambda_{a}=\left|\lambda_{a+1}\right|$, and $\mu_{a}=\left|\mu_{a+1}\right|$, in which case $h=1$.

Case III. $n$ is odd, $\mu=\lambda \pm e_{a}$ for some $a$, and $\lambda_{\ell}, \mu_{\ell}$ are not both zero. Then (case IIIa) $h=2$ unless (case IIIb) $\lambda_{\ell} \mu_{\ell}=0$, in which case $h=1$.

Proof. This is immediate from the selection rule (1.3). In case II, the order in which we add $\pm e_{a}$ and $\pm^{\prime} e_{b}$ is important when one move is needed to "clear" the other; that is, if $b=a+1$ and $\lambda_{a}=\left|\lambda_{b}\right|, \mu_{a}=\left|\mu_{b}\right|$. In case III, we get paths $\lambda \rightarrow \lambda \rightarrow \mu$ if $\lambda_{\ell} \neq 0$, and $\lambda \rightarrow \mu \rightarrow \mu$ if $\mu_{\ell} \neq 0$.

Second order leading symbols are in one-to-one correspondence with

$$
\operatorname{Hom}_{\operatorname{Spin}(n)}\left(\mathbb{S}^{2} M \otimes \mathbb{V}(\lambda), \mathbb{V}(\mu)\right),
$$

where $\mathbb{S}^{2} M$ is the bundle of covariant symmetric two-tensors. Thus only the part of $\operatorname{Hom}_{\operatorname{Spin}(n)}\left(T^{*} M \otimes T^{*} M \otimes \mathbb{V}(\lambda), \mathbb{V}(\mu)\right)$ corresponding to the $\mathbb{S}^{2}$ summand in $T^{*} M \otimes T^{*} M=\mathbb{S}^{2} M \oplus \Lambda^{2} M$ contributes to the symbol space.

Lemma 2.2. Let

$$
\begin{aligned}
& s=s_{\lambda \mu}:=\operatorname{dim} \operatorname{hom}_{\operatorname{Spin}(n)}\left(\mathbb{S}^{2} M \otimes \mathbb{V}(\lambda), \mathbb{V}(\mu)\right), \\
& t=t_{\lambda \mu}:=\operatorname{dim} \operatorname{hom}_{\operatorname{Spin}(n)}\left(\Lambda^{2} M \otimes \mathbb{V}(\lambda), \mathbb{V}(\mu)\right) .
\end{aligned}
$$

Then $s+t=h$; in particular, both $s$ and $t$ vanish except in cases I, II, III. In case I, $s=1$ and $t=0$. In cases IIa and IIIa, $s=t=1$. In cases IIb and IIIb, $s=0$ and $t=1$. Thus the dimension of the space of $\operatorname{Spin}(n)$-invariant second order leading symbols $\mathbb{V}(\lambda) \rightarrow \mathbb{V}(\mu)$ is 1 in cases I, IIa, IIIa, and 0 otherwise.

Proof. That $s+t=h$ is immediate from the decomposition $T^{*} M \otimes T^{*} M=\mathbb{S}^{2} M \oplus$ $\Lambda^{2} M$. We shall complete the proof by computing $s-t$.

Given two irreducible representations $\tau$ and $\lambda$ of a compact semisimple Lie algebra $\mathfrak{k}$, Kostant's formula [12] gives the decomposition into irreducibles of $\tau \otimes \lambda$ in terms of the highest weight of $\lambda$ and (all) the weights of $\tau$. In the case of interest here, weights are tuples in $\mathbb{Z}^{\ell} \cup\left(\frac{1}{2}+\mathbb{Z}\right)^{\ell}$. Let $\mathcal{W}$ be the Weyl group of $\mathfrak{s o}(n)$, which acts on weights by permutation and sign change of entries (arbitrarily many sign changes if $n$ is odd, an even number if $n$ is even). The $\operatorname{sign} \operatorname{sgn} w$ of $w \in \mathcal{W}$ is the 
sign of the permutation involved, times -1 to the number of sign changes. If $\alpha$ and $\beta$ are weights, let

$$
F_{\beta}(\alpha)=F_{\alpha}(\beta):=\sum_{w \in \mathcal{W}}(\operatorname{sgn} w) \delta_{w \beta}^{\alpha} .
$$

Let $q_{\tau}(\alpha)$ denote the multiplicity of $\alpha$ as a weight in $\tau$, and let $m_{\mu}(\tau \otimes \lambda)$ denote the multiplicity of an irreducible $\mu$ as a summand in $\tau \otimes \lambda$. Then

$$
\sum_{\alpha} q_{\tau}(\alpha) F_{\rho+\lambda-\alpha}=\sum_{\mu} m_{\mu}(\tau \otimes \lambda) F_{\rho+\mu} .
$$

Applying both sides of this equation to $\tilde{\mu}:=\rho+\mu$ for a given dominant weight $\mu$, we get

$$
m_{\mu}(\tau \otimes \lambda)=\sum_{\alpha} q_{\tau}(\alpha) F_{\rho+\lambda-\alpha}(\tilde{\mu})=\sum_{\alpha} q_{\tau}(\alpha) F_{\tilde{\mu}}(\rho+\lambda-\alpha),
$$

since the fact that $\tilde{\mu}$ is strictly dominant, $\tilde{\mu}_{1}>\ldots>\tilde{\mu}_{\ell-1}>\left|\tilde{\mu}_{\ell}\right|$, implies that only the identity in $\mathcal{W}$ can fix $\tilde{\mu}$. Both sides of (2.2) are additive in the $\sigma$ argument, so we can dispense with one of our irreducibility assumptions, and take $\sigma$ to be in the representation $\operatorname{ring} \mathcal{R}(\mathfrak{s o}(n))$.

We are interested in the situation in which $\tau=\mathbb{S}^{2} \ominus \Lambda^{2}$. The weights of $\mathbb{S}^{2}$ and $\Lambda^{2}$ are easily computed:

\begin{tabular}{|l|c|c|c|}
\hline \multicolumn{1}{|c|}{ weight $\alpha$} & $q_{\mathbb{S}^{2}}(\alpha)$ & $q_{\Lambda^{2}}(\alpha)$ & $q_{\mathbb{S}^{2} \ominus \Lambda^{2}}(\alpha)$ \\
\hline 0 & $n-l$ & $l$ & $n-2 l$ \\
\hline $2 e_{a}$ & 1 & 0 & 1 \\
\hline$-2 e_{a}$ & 1 & 0 & 1 \\
\hline$e_{a}-e_{b}, a \neq b$ & 1 & 1 & 0 \\
\hline$e_{a}+e_{b}, a \neq b$ & 1 & 1 & 0 \\
\hline$-e_{a}-e_{b}, a \neq b$ & 1 & 1 & 0 \\
\hline$e_{a}$ & $n-2 l$ & $n-2 l$ & 0 \\
\hline$-e_{a}$ & $n-2 l$ & $n-2 l$ & 0 \\
\hline
\end{tabular}

As a result,

$$
s-t=\sum_{a=1}^{\ell}\left\{F_{\tilde{\mu}}\left(\tilde{\lambda}+2 e_{a}\right)+F_{\tilde{\mu}}\left(\tilde{\lambda}-2 e_{a}\right)\right\} .
$$

We claim that at most one term in this sum is nonzero, and that for such a term $F_{\tilde{\mu}}\left(\tilde{\lambda} \pm 2 e_{a}\right)$, exactly one term in the sum (2.1) defining $F$ is nonzero.

Write $\alpha \sim \beta$ if $\alpha$ and $\beta$ are in the same $\mathcal{W}$-orbit. Note that if $\alpha \sim \beta$ and $\beta$ is strictly dominant, then $\left\{\left|\alpha_{a}\right| \mid 1 \leq a \leq \ell\right\}=\left\{\left|\beta_{a}\right| \mid 1 \leq a \leq \ell\right\}$ has $\ell$ distinct elements.

If $\tilde{\lambda}+2 e_{a} \sim \tilde{\mu} \sim \tilde{\lambda}-2 e_{a}$, we must have $\left|\tilde{\lambda}_{a}+2\right|=\left|\tilde{\lambda}_{a}-2\right|$, so $\tilde{\lambda}_{a}=0, a=\ell, n$ is even, and $\lambda_{\ell-1} \geq 2$. But then

$$
\tilde{\lambda}+2 e_{a}=\left(\tilde{\lambda}_{1}, \ldots, \tilde{\lambda}_{\ell-1}, 2\right) \nsim\left(\tilde{\lambda}_{1}, \ldots, \tilde{\lambda}_{\ell-1},-2\right)=\tilde{\lambda}-2 e_{a}
$$

since the number of negative entries does not agree. If $\tilde{\lambda} \pm 2 e_{a} \sim \tilde{\mu} \sim \tilde{\lambda} \pm^{\prime} 2 e_{b}$ for $a \neq b$, then $\left(\left|\tilde{\lambda}_{a} \pm 2\right|,\left|\tilde{\lambda}_{b}\right|\right)$ is a permutation of $\left(\left|\tilde{\lambda}_{a}\right|,\left|\tilde{\lambda}_{b} \pm^{\prime} 2\right|\right)$. If this permutation is the transposition, $\left|\tilde{\lambda}_{a}\right|=\left|\tilde{\lambda}_{b}\right|$, contradicting strict dominance. If the permutation is the identity, $\left|\tilde{\lambda}_{a}\right|=\left|\tilde{\lambda}_{b}\right|=1$, also a contradiction. Thus there is at most one contribution to (2.3). But since $\tilde{\mu}$ is strictly dominant, at most one term in (2.1) 
contributes to $F_{\tilde{\mu}}(\sigma)$ for any $\sigma$. This proves the claim. In particular, $|s-t| \leq 1$, so the lemma is proved in cases IIa, IIIa (where $s+t=h=2$ ). For the remaining cases, we just need to find the unique nonzero contribution to (2.1) and determine whether it is +1 or -1 .

In case I, the identity in $\mathcal{W}$ implements $\tilde{\lambda} \pm 2 e_{a} \sim \tilde{\mu}$, so $s-t=1$. In case IIb, if $\mu_{a}=\lambda_{a}+1$, then

$$
\tilde{\lambda} \pm^{\prime} 2 e_{a+1} \sim \tilde{\mu}
$$

provided the $\pm^{\prime}$ sign is chosen so that $\left|\tilde{\lambda}_{a+1} \pm^{\prime} 2\right|=\left|\tilde{\lambda}_{a+1}\right|+2$. The Weyl group element implementing (2.4) is a transposition together with 0 or 2 sign changes, so $s-t=\operatorname{sgn} w=-1$. If $\mu_{a}=\lambda_{a}-1$, then $\tilde{\lambda}_{a} \geq 2$, and $\tilde{\lambda}-2 e_{a} \sim \tilde{\mu}$ is again implemented by a transposition together with 0 or 2 sign changes; again $s-t=-1$. Finally, in case IIIb, $\left\{\lambda_{\ell}, \mu_{\ell}\right\}=\{0,1\}$, so $\left\{\tilde{\lambda}_{\ell}, \tilde{\mu}_{h}\right\}=\left\{\frac{1}{2}, \frac{3}{2}\right\}$, so $\tilde{\lambda}-2 e_{\ell} \sim \tilde{\mu}$ is implemented by a sign change, and again $s-t=-1$.

This means that there are second order $\operatorname{Spin}(n)$-operators in cases I, IIa, and IIIa. Recall that we are in the process of proving that there is a conformal covariant only in case I. The following lemma assures that one cannot form second-order conformal covariants for $\mu \neq \lambda$ by composing two gradients with compatible conformal weights.

Lemma 2.3. Suppose that $\lambda \neq \mu$ and $\sigma$ are dominant weights with $\lambda \rightarrow \sigma \rightarrow \mu$ under the selection rule (1.3), and that the reduced conformal biweights of $G_{\lambda \mu}, G_{\sigma \mu}$ are $(m, m+1)$ and $(m+1, m+2)$ respectively. Then we are in case IIb or IIIb, and the (conformally covariant) composition $G_{\sigma \mu} G_{\lambda \sigma}$ has vanishing second order symbol.

Proof. By Fegan's result (1.4), we must have

$$
m=\frac{1}{2}\{n-1+\langle\tilde{\lambda}+\tilde{\sigma}, \tilde{\lambda}-\tilde{\sigma}\rangle\}=\frac{1}{2}\{n-1+\langle\tilde{\sigma}+\tilde{\mu}, \tilde{\sigma}-\tilde{\mu}\rangle\}-1,
$$

so

$$
1=\frac{1}{2}\{\langle\tilde{\sigma}+\tilde{\mu}, \tilde{\sigma}-\tilde{\mu}\rangle-\langle\tilde{\lambda}+\tilde{\sigma}, \tilde{\lambda}-\tilde{\sigma}\rangle\} .
$$

Suppose first that $\lambda \neq \sigma \neq \mu$; then $\sigma=\lambda \pm e_{a}, \mu=\sigma \pm^{\prime} e_{b}$ for some $a, b$. (2.5) then becomes

$$
1= \pm \tilde{\sigma}_{a} \mp^{\prime} \tilde{\sigma}_{b} .
$$

If $\pm^{\prime}= \pm$, then $|a-b|=\left|\tilde{\sigma}_{a}-\tilde{\sigma}_{b}\right|=1, \sigma_{a}=\sigma_{b}$, and we are in case IIb, where by Lemma 2.2, there are no second order $\mathfrak{s o}(n)$-operators. If $\pm^{\prime}=\mp$, then $1=$ $\pm\left(\tilde{\sigma}_{a}+\tilde{\sigma}_{b}\right)$, so $\{a, b\} \subset\{\ell-1, \ell\}$; but $\mu \neq \lambda$ implies $a \neq b$, so $\{a, b\}=\{\ell-1, \ell\}$. Thus $n$ is even and $\sigma_{\ell}=-\sigma_{\ell-1} \leq 0$. But then we are in case IIb again.

It remains to consider the cases $\sigma=\lambda$ and $\sigma=\mu$ (in which $n$ must be odd). These cases are dual: $\left(G_{\lambda \sigma}, G_{\sigma \mu}\right)$ is a pair of the type we seek if and only if $\left(G_{\mu \sigma}, G_{\sigma \lambda}\right) \cong_{\mathfrak{s o}(n)}\left(G_{\sigma \mu}^{*}, G_{\lambda \sigma}^{*}\right)$ is. Thus we need only treat the case $\lambda=\sigma \neq \mu$; say $\mu=\sigma \pm e_{a}$. But then (2.5) gives $1=\mp\left(2 \tilde{\sigma}_{a} \pm 1\right) / 2$, i.e. $\tilde{\sigma}_{a}=\mp \frac{3}{2}$. Thus $\pm=-$ and $a$ is $\ell-1$ or $\ell$. If $a=\ell-1$, then $\sigma_{\ell-1}=\sigma_{\ell}=0$, contradicting $\sigma \rightarrow \sigma$ (or $\mu=\sigma-e_{\ell}$ ). If $a=\ell$, then $\sigma_{\ell}=1$ and $\mu_{\ell}=0$, and we are in case IIIb, where by Lemma 2.2 , there are no second order $\mathfrak{s o}(n)$-operators.

Remark 2.4. In any tensor-spinor realization, Weyl's invariant theory [16] shows that the operator $D=G_{\sigma \mu} G_{\lambda \sigma}$ considered in Lemma 2.3 is polynomial in the 
covariant derivative $\nabla$, the Riemann tensor $R$, the metric tensor $g$ and its inverse, the volume form $E$, and the fundamental tensor-spinor $\gamma$. The dilational behavior $g^{\prime}=A^{2} g, A \in \mathbb{R}_{+} \Rightarrow D^{\prime}=A^{-2} D$ shows that each monomial term in this polynomial has $k_{\nabla}+2 k_{R}=2$, where $k_{\nabla}$ is the number of explicit occurrences of $\nabla$, and similarly for $k_{R}$. This, together with the vanishing of the second order symbol of $D$, shows that ord $D=0$. Now the Weyl conformal curvature tensor is

$$
C^{i}{ }_{j k l}=R^{i}{ }_{j k l}+V_{j k} \delta^{i}{ }_{l}-V_{j l} \delta^{i}{ }_{k}+V^{i}{ }_{l} g_{j k}-V^{i}{ }_{k} g_{j l},
$$

where $V$ is the normalized Ricci tensor of (1.7). $C$ is conformally invariant, and the conformal behavior of $V$ is as follows: if $g_{\varepsilon \omega}=e^{2 \varepsilon \omega} g_{0}$ is a conformal curve of metrics, $\varepsilon$ running through a real interval about 0 and $\omega$ being a $C^{\infty}$ function, then

$$
\left.(d / d \varepsilon)\right|_{\varepsilon=0} V=-\nabla \nabla \omega .
$$

Since the operator $D$ studied in Lemma 2.3 is conformally covariant, it must be an action of $C$ : it has the form $\varphi \mapsto \alpha(C \otimes \varphi)$, where $\alpha \in \operatorname{Hom}_{\mathfrak{s o}(n)}(\mathcal{C} \otimes \mathbb{V}(\lambda), \mathbb{V}(\mu))$.

In view of Remark 2.4, our only chance to get a conformal covariant $\mathbb{V}(\lambda) \rightarrow \mathbb{V}(\mu)$ is as follows. In cases where $\operatorname{Hom}_{\mathfrak{s o}(n)}\left(\mathbb{S}^{2} M \otimes \mathbb{V}(\lambda), \mathbb{V}(\mu)\right)$ is 1-dimensional, we attempt to make a "curvature correction" to the operator $\operatorname{Proj}_{\mathbb{V}(\mu)}(\nabla \nabla)$ on $\mathbb{V}(\lambda)$. By the conformal covariance of $C$, only corrections by actions of $V$ can alter the operator's conformal behavior. Such an action has the form $\varphi \mapsto \beta(V \otimes \varphi)$, where $\beta \in \operatorname{Hom}_{\mathfrak{s o}(n)}\left(\mathbb{S}^{2} M \otimes \mathbb{V}(\lambda), \mathbb{V}(\mu)\right)$, since $V$ is a section of $\mathbb{S}^{2} M$. By Lemma 2.2, this Hom space has dimension at most 1 , so our prospective conformal covariant must have the form

$$
\operatorname{Proj}_{\mathbb{V}(\mu)}(\nabla \nabla+c V \otimes), \quad c \in \mathbb{R} .
$$

We need to show that there is a unique $c$ for which (2.7) is conformally covariant in Case I, and no such $c$ in Cases IIa and IIIa. To handle Case I, first note that the conformal covariance condition (1.1) is equivalent to its infinitesimal form [5, Proposition 1.12]

$$
\left.(d / d \varepsilon)\right|_{\varepsilon=0} D=-(b-a) \omega D+a[D, \operatorname{mult}(\omega)],
$$

where $\left.(d / d \varepsilon)\right|_{\varepsilon=0}$ is the conformal variation described in Remark 2.4, and "mult" means "multiplication by".

In case I, $\lambda \rightarrow \sigma:=\lambda+\varepsilon e_{a} \rightarrow \mu$ is the only selection rule path from $\lambda$ to $\mu$. Thus

$$
\left.\operatorname{Proj}_{\mathbb{V}(\mu)} \nabla \nabla\right|_{\mathbb{V}(\lambda)}=G_{\sigma \mu} G_{\lambda \sigma}
$$

By (1.4), $G_{\lambda \sigma}$ has reduced conformal biweight $(m+1, m+2)$, where $m=\frac{1}{2}\left(n-2 \varepsilon \tilde{\mu}_{a}\right)$, and $G_{\sigma \mu}$ has conformal biweight $(m, m+1)$. Thus the conformal variation of $G_{\sigma \mu} G_{\lambda \sigma}$, viewed as an operator from $\mathbb{V}^{m}(\lambda)$ to $\mathbb{V}^{m+2}(\mu)$, is

$$
\begin{aligned}
\left.(d / d \varepsilon)\right|_{\varepsilon=0}\left(G_{\sigma \mu} G_{\lambda \sigma}\right) & =\left.G_{\sigma \mu}(d / d \varepsilon)\right|_{\varepsilon=0} G_{\lambda \sigma}+\left(\left.(d / d \varepsilon)\right|_{\varepsilon=0} G_{\sigma \mu}\right) G_{\lambda \sigma} \\
& =G_{\sigma \mu}\left[G_{\lambda \sigma}, \operatorname{mult}(\omega)\right]-\left[G_{\sigma \mu}, \operatorname{mult}(\omega)\right] G_{\lambda \sigma} \\
& =G_{\sigma \mu} \zeta_{\lambda \sigma}(d \omega)-\zeta_{\sigma \mu}(d \omega) G_{\lambda \sigma} \\
& =\left.\operatorname{Proj}_{\mathbb{V}(\mu)}[\nabla, \tau(d \omega)]\right|_{\mathbb{V}(\lambda)}
\end{aligned}
$$


where, for a one-form $\eta, \tau(\eta) \varphi:=\eta \otimes \varphi$, and

$$
\zeta_{\lambda \sigma}(\nu):=\left.\operatorname{Proj}_{\mathbb{V}(\sigma)} \tau(\eta)\right|_{\mathbb{V}(\lambda)} .
$$

But $[\nabla, \tau(\eta)] \varphi=(\nabla \eta) \otimes \varphi$, whence

$$
\left(\left.(d / d \varepsilon)\right|_{\varepsilon=0}\left(G_{\sigma \mu} G_{\lambda \sigma}\right)\right) \varphi=\operatorname{Proj}_{\mathbb{V}(\mu)}(\nabla \nabla \omega) \otimes \varphi .
$$

By this, (2.6), and the conformal invariance of $\operatorname{Proj}_{\mathbb{V}(\mu)}$, the operator $D_{\lambda \mu}$ described in the statement of Theorem 1.2 is the unique conformal covariant $\mathbb{V}(\lambda) \rightarrow \mathbb{V}(\mu)$ of reduced biweight $(m, m+2)$.

For uniqueness, we still have to prove that no operator $D$ of the form (2.7) has a different reduced conformal biweight $\left(m^{\prime}, m^{\prime}+2\right)$. Viewing $D$ as an operator $\mathbb{V}^{m}(\lambda) \rightarrow \mathbb{V}^{m+2}(\mu)$, we get

$$
\left(\left.(d / d \varepsilon)\right|_{\varepsilon=0} D\right) \varphi=\left(\left.(d / d \varepsilon)\right|_{\varepsilon=0}\left(D-D_{\lambda \mu}\right)\right) \varphi=(c-1)(\nabla \nabla \omega) \otimes \varphi .
$$

But the putative conformal behavior of $D$ gives

$$
\left.(d / d \varepsilon)\right|_{\varepsilon=0} D=\left(m^{\prime}-m\right)[D, \operatorname{mult}(\omega)]=\operatorname{Proj}_{\mathbb{V}(\mu)}[\nabla \nabla, \operatorname{mult}(\omega)],
$$

an operator with nonvanishing first order leading symbol for nonconstant $\omega$. This contradicts (2.8).

In cases IIa and IIIa, there are dominant weights $\sigma$ and $\tau$ for which

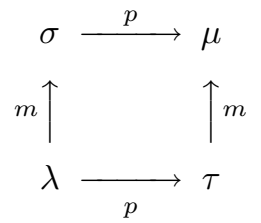

is the complete list of second order paths from $\lambda$ to $\mu$. Here $m$ and $p$ are distinct, $G_{\lambda \sigma}$ and $G_{\tau \mu}$ both have reduced conformal biweight $(m, m+1)$, and $G_{\sigma \mu}$ and $G_{\lambda \tau}$ both have reduced conformal biweight $(p, p+1) . s_{\lambda \mu}=t_{\lambda \mu}=1$ in both cases, so $\operatorname{ord}\left(a G_{\sigma \mu} G_{\lambda \sigma}+b G_{\tau \mu} G_{\lambda \tau}\right)=0$ for some $(0,0) \neq(a, b) \in \mathbb{R}^{2}$. In particular,

$$
\operatorname{ord}\left(\left.(d / d \varepsilon)\right|_{\varepsilon=0}\left(a G_{\sigma \mu} G_{\lambda \sigma}+b G_{\tau \mu} G_{\lambda \tau}\right)\right)=0
$$

whenever the operator $a G_{\sigma \mu} G_{\lambda \sigma}+b G_{\tau \mu} G_{\lambda \tau}$ is viewed as carrying $\mathbb{V}^{q}(\lambda)$ to $\mathbb{V}^{q+2}(\mu)$, for any $q$. Suppose, to obtain a contradiction, that there is a second order conformal covariant $D: \mathbb{V}(\lambda) \rightarrow \mathbb{V}(\mu)$; let its reduced biweight be $\left(q_{0}, q_{0}+2\right)$. D must have the same leading symbol as $\alpha G_{\sigma \mu} G_{\lambda \sigma}+\beta G_{\tau \mu} G_{\lambda \tau}$ for some $(\alpha, \beta) \in \mathbb{R}^{2}$ linearly independent of $(a, b)$. Viewing our operators as carrying $\mathbb{V}^{q_{0}}(\lambda) \rightarrow \mathbb{V}^{q_{0}+2}(\mu)$, we have (2.10) with $\alpha, \beta$ in place of $a, b$. Since $\sqrt{-1} \zeta_{\lambda \sigma}$ is the leading symbol of $G_{\lambda \sigma}$,

$$
\begin{aligned}
0= & \sigma_{1}\left(\left.(d / d \varepsilon)\right|_{\varepsilon=0}\left(G_{\sigma \mu} G_{\lambda \sigma}\right)\right)(\xi) \\
= & \sqrt{-1}\left\{\zeta_{\sigma \mu}(\xi)\left(q_{0}-m\right)\left[G_{\lambda \sigma}, \operatorname{mult}(\omega)\right]+\left(q_{0}+1-p\right)\left[G_{\sigma \mu}, \operatorname{mult}(\omega)\right] \zeta_{\lambda \sigma}(\xi)\right\} \\
= & \sqrt{-1}\left\{\left(q_{0}+\frac{1}{2}(1-m-p)\right)\left(\zeta_{\sigma \mu}(\xi) \zeta_{\lambda \sigma}(d \omega)+\zeta_{\sigma \mu}(d \omega) \zeta_{\lambda \sigma}(\xi)\right)\right. \\
& \left.\quad+\frac{1}{2}(p-m-1)\left(\zeta_{\sigma \mu}(\xi) \zeta_{\lambda \sigma}(d \omega)-\zeta_{\sigma \mu}(d \omega) \zeta_{\lambda \sigma}(\xi)\right)\right\}
\end{aligned}
$$

Similarly,

$$
\begin{aligned}
0= & \sigma_{1}\left(\left.(d / d \varepsilon)\right|_{\varepsilon=0}\left(G_{\tau \mu} G_{\lambda \tau}\right)\right)(\xi) \\
= & \sqrt{-1}\left\{\left(q_{0}+\frac{1}{2}(1-p-m)\right)\left(\zeta_{\tau \mu}(\xi) \zeta_{\lambda \tau}(d \omega)+\zeta_{\tau \mu}(d \omega) \zeta_{\lambda \tau}(\xi)\right)\right. \\
& \left.\quad+\frac{1}{2}(m-p-1)\left(\zeta_{\tau \mu}(\xi) \zeta_{\lambda \tau}(d \omega)-\zeta_{\tau \mu}(d \omega) \zeta_{\lambda \tau}(\xi)\right)\right\}
\end{aligned}
$$


To avoid contradicting $t_{\lambda \mu}=1$, we must have $p=m \pm 1$; but by Lemma 2.3 and (2.9), this can only happen in cases IIb and IIIb. Thus there can be no conformal covariant in cases IIa and IIIa; this completes the proof of Theorem 1.2.

\section{REMARKS AND EXAMPLES}

Remark 3.1. Recall that in Theorem 1.1 and Theorem 1.2, we work modulo actions of the Weyl tensor, summands as in formula (1.2) arising from elements $\alpha$ of $\operatorname{Hom}_{\mathfrak{s o}(n)}(\mathcal{C} \otimes \mathbb{V}(\lambda), \mathbb{V}(\mu))$. Using formulas of Freudenthal [10] and of Brauer [8], it is straightforward to count such actions; i.e. to determine $\operatorname{dim} \operatorname{Hom}_{\mathfrak{s o}(n)}(\mathcal{C} \otimes$ $\mathbb{V}(\lambda), \mathbb{V}(\mu)$ ). (Note that the question makes sense only for $n \geq 4$, since there is no Weyl tensor in dimension 3.) In [3], we count the Weyl tensor actions in the case

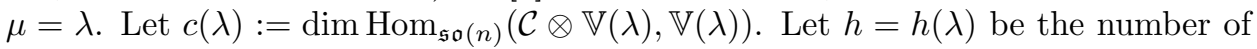
steep drops in $\lambda$, i.e. indices $a$ with $\lambda_{a}>\left|\lambda_{a+1}\right|+1$; let $r=r(\lambda)$ be the number of distinct entries in the $\ell$-tuple $\lambda$, and let $s=s(\lambda)$ be the number of distinct entries appearing at least twice in $\lambda$. Then if $\lambda_{\ell} \geq 0$,

$$
c(\lambda)=\left\{\begin{array}{l}
(r-1)^{2}+s+h-r \delta_{(0,0)}^{\left(\lambda_{\ell-1}, \lambda_{\ell}\right)}-\delta_{\left(\frac{1}{2}, \frac{1}{2}\right)}^{\left.\left(\lambda_{\ell-1}\right), \lambda_{\ell}\right)}, n \geq 4 \text { even, } \\
r(r-1)+s+h-2(r-1) \delta_{0}^{\lambda_{\ell}}-\delta_{(0,0)}^{\left(\lambda_{\ell-1}, \lambda_{\ell}\right)}-(r-1) \delta_{1 / 2}^{\lambda_{\ell}}-\delta_{\left(\frac{1}{2}, \frac{1}{2}\right)}^{\left(\lambda_{\ell-1}, \lambda_{\ell}\right)}, \\
\quad n \geq 5 \text { odd. }
\end{array}\right.
$$

If $\lambda_{\ell}<0$, then $c(\lambda)=c\left(\lambda_{1}, \ldots, \lambda_{\ell-1},-\lambda_{\ell}\right)$. When $n=4$, we can distinguish the contributions of $C_{ \pm}$, the self- and anti-self-dual parts of $C$. If $\lambda_{2} \geq 0$, then

$$
c_{+}(\lambda)=1-r \delta_{(0,0)}^{\left(\lambda_{1}, \lambda_{2}\right)}-\delta_{\left(\frac{1}{2}, \frac{1}{2}\right)}^{\left(\lambda_{1}, \lambda_{2}\right)}, \quad c_{-}(\lambda)=h .
$$

(Note that $(r-1)^{2}+s=1$ in this case, since $(r, s)$ is either $(2,0)$ or $(1,1)$.) If $\lambda_{2}<0$, then $c_{ \pm}(\lambda)=c_{\mp}\left(\lambda_{1},-\lambda_{2}\right)$.

Remark 3.2. The conformal covariants $D_{\lambda, \lambda+2 e_{a}}$ and $D_{\lambda+2 e_{a}, \lambda}$ (for $\lambda, \lambda+2 e_{a}$ both dominant) are formal adjoints of each other.

Remark 3.3. The operator (1.6) is unchanged upon replacing $V$ with its trace free part $B=V-J g / n$ (where $J=V^{i}{ }_{i}=K / 2(n-1)$ ), since $\mathbb{S}^{2}$ splits as $\operatorname{TFS}^{2} \oplus \Lambda^{0}$, where $\mathrm{TFS}^{2}$ is the bundle of trace free symmetric two-tensors. Since $\Lambda^{0} \otimes \mathbb{V}(\lambda)=$ $\mathbb{V}(\lambda)$, we "cannot reach" $\mathbb{V}(\mu)$ by tensoring with the $\Lambda^{0}$ summand when $\mu \neq \lambda$.

Example 3.4. In [4], the following conformal covariant on the $k$-form bundle $\Lambda^{k}$ was introduced: let $s=(n-2 k) / 2$; then

$$
D_{k}=(s+1) \delta d+(s-1) d \delta+(s+1)(s-1)(J-2 V \cdot),
$$

where $V$. is the natural (derivation) action of the 2-tensor $V$ on the Grassmann algebra. $D_{0}$ is the conformal Laplacian, and $D_{(n-2) / 2}=\delta d$ is the Maxwell operator on vector potentials for $n$ even. For $k \neq n / 2$, this operator is a special case of that given in Theorem 1.1. (The Ricci tensor action apparent in (3.2) is hidden in the peculiar combination of gradients in (1.5); see [6, Example 5.7]. When $n$ is even and $k=n / 2$, we have two operators of the type described in Theorem 1.2. Indeed, (3.2) anticommutes with the Hodge $\star$ operator:

$$
\star D_{k}=-D_{n-k} \star .
$$


As a result, $D_{n / 2}$ interchanges the two eigenbundles $\mathbb{V}(1, \ldots, 1, \pm 1)$ of $\star$ on middleforms. Choose a complex unit $v$ with square $(-1)^{n / 2}$, and denote by $\Lambda_{ \pm}^{n / 2}$ the $\pm v$ eigenbundle of $\star$. Then the $D_{ \pm}: \Lambda_{ \pm}^{\ell} \rightarrow \Lambda_{\mp}^{\ell}$ are

$$
D_{ \pm}=G_{\mp}^{*} G_{ \pm}+B_{i j} \zeta_{\mp}\left(d x^{i}\right)^{*} \zeta_{ \pm}\left(d x^{j}\right),
$$

where $G_{ \pm}$is the gradient with target $\mathbb{V}\left(1_{\ell-1}\right)$, and $\sqrt{-1} \zeta_{ \pm}$is its leading symbol. We first claim that the copy $X_{ \pm}$of the bundle $\mathbb{V}\left(1_{\ell-1}\right)$ which is involved is cut out of $\Lambda^{\ell+1} \oplus \Lambda^{\ell-1}$ by the condition

$$
\operatorname{Proj}_{\Lambda^{\ell-1}} \psi=\mp v \star \operatorname{Proj}_{\Lambda^{\ell+1}} \psi .
$$

For if $\eta$ is a one-form and $\varphi$ a section of $\Lambda_{ \pm}^{\ell}$, calculations like those in [6, Example 5.7] give

$$
\operatorname{Proj}_{\Lambda^{\ell+1} \oplus \Lambda^{\ell-1}}(\eta \otimes \varphi)=(\ell+1)^{-1}(\varepsilon(\eta)-\iota(\eta)) \varphi,
$$

where $\varepsilon$ and $i$ are exterior and interior multiplication. But $\iota(\eta) \varphi=\star \varepsilon(\eta) \star \varphi=$ $v \star \varepsilon(\eta) \varphi$. (The evenness of $n$ is used in the identity $\iota=\star \varepsilon \star$.) The complementary projections $P_{ \pm}$of $\Lambda^{\ell+1} \oplus \Lambda^{\ell-1}$ onto $X_{ \pm}$are thus

$$
P_{ \pm} \psi=\frac{1}{2}(\psi \mp v \star \psi) \text {. }
$$

The bundle maps

$$
I_{ \pm}=2 \operatorname{Proj}_{\Lambda^{\ell-1}}: X_{ \pm} \rightarrow \Lambda^{\ell-1}
$$

are natural isometries with $\Lambda^{\ell-1}$, which we may view as a "standard realization" of $\mathbb{V}\left(1_{\ell-1}\right)$. Using calculations from [6, Example 5.7] to set normalizations, we have

$$
\begin{aligned}
D_{ \pm} & =(\delta+d) P_{\mp} I_{ \pm}(\ell+1)^{-1}(\delta+d)+B_{i j}(\iota-\varepsilon)\left(d x^{i}\right) P_{\mp} I_{ \pm}(\ell+1)^{-1}(\varepsilon-\iota)\left(d x^{j}\right) \\
& =(\ell+1)^{-1}\left\{(\delta+d)(\delta \pm v \star \delta)-B_{i j}(\iota-\varepsilon)\left(d x^{i}\right)(\iota \pm v \star \iota)\left(d x^{j}\right)\right\} .
\end{aligned}
$$

But $v \star \delta \varphi=-v \star \star d( \pm v \varphi)=\mp d \varphi$ and $v \star \iota\left(d x^{j}\right) \varphi=v \star \star \varepsilon\left(d x^{j}\right)( \pm v) \varphi= \pm \varepsilon\left(d x^{j}\right) \varphi$, so

$$
\begin{aligned}
-(\ell+1) D_{ \pm} & =-(\delta+d)(\delta-d)+B_{i j}(\iota-\varepsilon)\left(d x^{i}\right)(\iota+\varepsilon)\left(d x^{j}\right) \\
& =\delta d-d \delta-2 B_{i j} \varepsilon\left(d x^{i}\right) \iota\left(d x^{j}\right) \\
& =\delta d-d \delta-2 B .
\end{aligned}
$$

since $\varepsilon\left(d x^{i}\right) \varepsilon\left(d x^{j}\right)=\iota\left(d x^{i}\right) \iota\left(d x^{j}\right)=0$ and $\iota\left(d x^{i}\right) \varepsilon\left(d x^{j}\right)+\varepsilon\left(d x^{j}\right) \iota\left(d x^{i}\right)=g^{i j}$. Since $B=V-J g / n$ and $\left.g \cdot\right|_{\Lambda^{k}}=k$, this is exactly the operator $\delta d-d \delta-2 V \cdot+J$ of (3.2).

Example 3.5. The bundle $\mathrm{TFS}^{p}$ of trace free symmetric $p$-tensors is $\mathbb{V}(p, 0, \ldots, 0)$. To get a tensor realization of the conformal covariant carrying $\mathbb{V}(p, 0, \ldots, 0)$ to $\mathbb{V}(p+2,0, \ldots, 0)$, note first that a formula for $\operatorname{Proj}_{\mathrm{TFS}^{p+1}}: \Lambda^{1} \otimes \mathrm{TFS}^{p} \rightarrow \mathrm{TFS}^{p+1}$ is given by

$$
\left(\operatorname{Proj}_{\mathrm{TFS}^{p+1}} \varphi\right)_{i_{1} \ldots i_{p+1}}=\varphi_{\left(i_{1} \ldots i_{p+1}\right)}-a \varphi_{l\left(i_{1} \ldots i_{p-1}\right.}^{l} g_{\left.i_{p} i_{p+1}\right)},
$$

where $a$ is a constant, and the indices in parentheses are to be symmetrized over. To make the $i_{1} i_{2}$ trace (and thus all traces) vanish, we need

$$
a=p /(n+2 p-2) \text {. }
$$


Iterating (3.2) and (3.3), we find that $\operatorname{Proj}_{\mathrm{TFS}^{p+2}}: \Lambda^{1} \otimes \Lambda^{1} \otimes \mathrm{TFS}^{p} \rightarrow \mathrm{TFS}^{p+2}$ is given by

$$
\begin{gathered}
\left(\operatorname{Proj}_{\mathrm{TFS} p+2} \varphi\right)_{i_{1} \ldots i_{p+2}}=\varphi_{\left(i_{1} \ldots i_{p+2}\right)}-\frac{p}{n+2 p} \operatorname{Symm}\left(\varphi_{i_{1} l i_{2} \ldots i_{p}}^{l}+\varphi_{i_{1}}{ }_{l i_{2} \ldots i_{p}}\right) g_{i_{p+1} i_{p+2}} \\
-\frac{1}{n+2 p} \varphi_{l\left(i_{1} \ldots i_{p}\right.}^{l} g_{\left.i_{p+1} i_{p+2}\right)}+\frac{p(p-1)}{(n+2 p)(n+2 p-2)} \varphi^{l m}{ }_{l m\left(i_{1} \ldots i_{p-2}\right.} g_{i_{p-1} i_{p}} g_{\left.i_{p+1} i_{p+2}\right)},
\end{gathered}
$$

where "Symm" is symmetrization over $i_{1} \ldots i_{p+2}$. If $\varphi$ is a section of $\mathrm{TFS}^{2} \otimes \mathrm{TFS}^{p}$, this simplifies somewhat:

$$
\begin{aligned}
& \left(\operatorname{Proj}_{\mathrm{TFS}^{p+2}} \varphi\right)_{i_{1} \ldots i_{p+2}}=\varphi_{\left(i_{1} \ldots i_{p+2}\right)}-\frac{2 p}{n+2 p} \operatorname{Symm} \varphi_{i_{1} l i_{2} \ldots i_{p}}^{l} g_{i_{p+1} i_{p+2}} \\
& \quad+\frac{p(p-1)}{(n+2 p)(n+2 p-2)} \varphi_{l m\left(i_{1} \ldots i_{p-2}\right.}^{l m} g_{i_{p-1} i_{p}} g_{\left.i_{p+1} i_{p+2}\right)} .
\end{aligned}
$$

Let $\mathcal{D}_{i j}=\frac{1}{2}\left(\nabla_{i} \nabla_{j}+\nabla_{j} \nabla_{i}\right)-\frac{1}{n} g_{i j} \nabla^{l} \nabla_{l}$. By (1.6) and (3.4), our conformal covariant is

$$
\begin{aligned}
P_{p}:= & (\mathcal{D}+B)_{\left(i_{1} i_{2}\right.} \varphi_{\left.i_{3} \ldots i_{p+2}\right)}-\frac{2 p}{n+2 p} \operatorname{Symm}(\mathcal{D}+B)^{l}{ }_{i_{1}} \varphi_{l i_{2} \ldots i_{p}} g_{i_{p+1} i_{p+2}} \\
& +\frac{p(p-1)}{(n+2 p)(n+2 p-2)}(\mathcal{D}+B)^{l m} \varphi_{l m\left(i_{1} \ldots i_{p-2}\right.} g_{i_{p-1} i_{p}} g_{\left.i_{p+1} i_{p+2}\right)} .
\end{aligned}
$$

Taking formal adjoints,

$$
\left(P_{p}^{*} \psi\right)_{i_{1} \ldots i_{p}}=(\mathcal{D}+B)^{l m} \psi_{l m i_{1} \ldots i_{p}}=\left(\nabla^{l} \nabla^{m}+V^{l m}\right) \psi_{l m i_{1} \ldots i_{p}} .
$$

The $P_{p}$ and $P_{p}^{*}$ were first introduced, in an ad hoc calculation that applies only to the $\mathrm{TFS}^{p}$ bundles, in $[11, \S 3]$.

\section{REFERENCES}

1. R. Baston, Verma modules and differential conformal invariants, J. Diff. Geom. 32 (1990), 851-898. MR 92a:58151

2. R. Baston and M. Eastwood, Invariant operators, Twistors in Mathematics and Physics, London Mathematical Society Lecture Notes, vol. 156, Cambridge University Press, 1990, pp. 129-163. MR 92b:58247

3. C. Bennett and T. Branson, Curvature actions, in preparation.

4. T. Branson, Conformally covariant equations on differential forms, Comm. Partial Differential Equations 7 (1982), 393-431. MR 84g:58110

5. T. Branson, Differential operators canonically associated to a conformal structure, Math. Scand. 57 (1985), 293-345. MR 88a:58212

6. T. Branson, Nonlinear phenomena in the spectral theory of geometric linear differential operators, Proc. Symp. Pure Math. 59 (1996), 27-65. CMP 96:13

7. T. Branson, Stein-Weiss operators and ellipticity, J. Funct. Anal., to appear.

8. R. Brauer, Sur la multiplication des caractéristiques des groupes continus et semi-simples, C.R. Acad. Sci. Paris 204 (1937), 1784-1786.

9. H. D. Fegan, Conformally invariant first order differential operators, Quart. J. Math. Oxford 27 (1976), 371-378. MR 58:2920

10. H. Freudenthal, Zur Berechnung der Charaktere der halbeinfachen Lieschen Gruppen, I,II, Indag. Math. 16 (1954), 369-376 and 487-491. MR 16:673a

11. R. Jenne, A construction of conformally invariant differential operators, Ph.D. dissertation, University of Washington, 1988.

12. B. Kostant, A formula for the multiplicity of a weight, Trans. Amer. Math. Soc. 93 (1959), 53-73. MR 22:80

13. S. Paneitz, A quartic conformally covariant differential operator for arbitrary pseudo-Riemannian manifolds, preprint, 1983. 
14. J. Slovák, Invariant operators on conformal manifolds, lecture notes, University of Vienna, 1992, archived at file://ftp.math.muni.cz.

15. E. Stein and G. Weiss, Generalization of the Cauchy-Riemann equations and representations of the rotation group, Amer J. Math. 90 (1968), 163-196. MR 36:6540

16. H. Weyl, The Classical Groups: Their Invariants and Representations, Princeton University Press, Princeton, 1939.

17. V. Wünsch, On conformally invariant differential operators, Math. Nachr. 129 (1986), 269281. MR 88a:58207

Department of Mathematics, The University of Iowa, Iowa City, Iowa 52242

E-mail address: branson@math.uiowa.edu 